\title{
\begin{tabular}{|l|l|l|l|l|}
\hline $\mathrm{M}$ & $\mathrm{R}$ & $\mathrm{S}$ & Internet Journal of & Nitride Semiconductor Research \\
\hline
\end{tabular}
}

Volume 1, Article 19

\section{Microstructure, growth mechanisms and electro-optical properties of heteroepitaxial GaN layers on sapphire (0001) substrates}

\author{
S. Christiansen, M. Albrecht, W. Dorsch, H. P. Strunk \\ Institut für Werkstoffwissenschaften, Lehrstuhl VII, Universität Erlangen-Nürnberg \\ C. Zanotti-Fregonara, G. Salviati \\ CNR-MASPEC Institute \\ A. Pelzmann, M. Mayer, Markus Kamp , KJ Ebeling \\ Abteilung Optoelektronik, Universität UIm
}

This article was received on June 11, 1996 and accepted on October 22, 1996.

\begin{abstract}
We investigate the structure, growth morphology and the related electro-optical properties of gallium nitride (GaN) films deposited on (0001) sapphire substrates by gas source molecular beam epitaxy (GSMBE) and use transmismission electron microscopy, atomic force microscopy and scanning tunneling microscopy, photoluminescence $(\mathrm{PL})$ and cathodoluminescence $(\mathrm{CL})$. We find two types of specimens: one type which shows a strong UV luminescence (band-to-band transition at 358nm/3.46eV) in CL and PL and only faint yellow luminescence (Gaussian shaped CL/PL peaks at around $528 \mathrm{~nm} / 2.35 \mathrm{eV}$ ), specimen 'B', and another type, which shows a strong UV and a comparably strong yellow luminescence, specimen ' $Y$ '. These two types of specimens have a rough layer surface, specimen ' $Y$ ' even an islanded one with, facetted hexagonal islands with a width of $1-2 \mu \mathrm{m}$ at a height of $50 \mathrm{~nm}$. A correlation of spectrally resolved CL images to the observed defect structure shows: (i) the yellow luminescence is homogeneously distributed over the complete specimen for ' $B$ ' and ' $Y$ ' specimens. Our investigations strongly suggest the yellow luminescence to be related to screw dislocations with $\vec{b}=[0001]$, which are found randomly distributed in ' $\mathrm{B}$ ' and ' $\mathrm{Y}$ ' specimens with a high density of $1.3 \cdot 10^{9} \mathrm{~cm}^{-2}$; (ii) the strong UV luminescence in ' $Y$ ' specimens is located in the troughs between adjacent surface islands, where dislocations essentially in small angle grain boundaries of edge type, i.e. with $\vec{b}=[\overline{1} 2 \overline{1} 0]$ or $\vec{b}=[\overline{1} \overline{1} 23]$ are located; (iii) in the case of the 'B' specimens these dislocations are randomly distributed and so is the luminescence.
\end{abstract}

\section{Introduction}

Today $\alpha-G a N$ (hexagonal polytype with wurzite structure) can be deposited successfully by metalorganic chemical vapor deposition (MOVPE) [1] [2] [3] as well as molecular beam epitaxial (MBE) methods [4] [5] [6]. It is now the timely task to correlate differences in electro-optical properties (i.e. photoluminescence (PL), cathodoluminescence $(C L))$ of those films to differences in the micro-and defect structure. While in high quality $\alpha$-GaN layers only band-to-band luminescence at $358 \mathrm{~nm} / 3.46 \mathrm{eV}$, which is responsible for the UV light emission [7] [8] [9] is observed, in lower quality material in addition a so-called yellow luminescence at about $528 \mathrm{~nm} / 2.35 \mathrm{eV}$ [9] [10] [11] [12] occurs.

It is generally accepted (cf. [12]), that the undefected volume of $\alpha-G a N$ is responsible for the UV luminescence, while the origin of the yellow luminescence which acts as a lifetime killer and suppresses the desired UV luminescence is still under discussion. Even the origin of the related recombination mechanism is still controversal (Is it a transition from a shallow donor to a deep acceptor [10] or from a deep donor to a shallow acceptor [13]?). It is however self evident that a crystal defect must be involved, which has up to now not been identified. Ponce et al. [9] proposed, based upon the correlation of CL measurements and TEM defect analysis, that small angle grain boundaries between small crystallites, which originate from columnar growth or point defects which nucleate at 
these dislocations [9] contribute to the yellow luminescence. Singh et al. [14] suggested intrinsic (point) defects to be responsible for the yellow luminescence.

We in this paper correlate PL and CL measurements with a thorough study of the defect structure by means of high resolution (HRTEM) and conventional transmission electron microscopy (TEM) (in plane view and cross-section) and thus complement Ponces' findings [9]. To this end we compare two essentially different types of samples, in the following referred to as sample ' $Y$ ' and ' $B$ ':

1. ' $Y$ ' specimens show a strong yellow luminescence,

2. 'B' specimens only a faint one, but a strong UV luminescence

(cf. typical PL data, obtained at 300K shown in Figure 1).

\section{Experimental}

The $\alpha$-GaN films under study are grown on (0001) sapphire substrates by GSMBE (for details of the deposition method see [4] [5]). The $\alpha$-GaN films of sample ' $Y$ ' are grown at $876^{\circ} \mathrm{C}$ to a total thickness of $1.7 \mu \mathrm{m}$ directly on epi-ready polished sapphire substrates, the $\alpha-G a N$ films of sample 'B' are grown at lower fluxes under otherwise identical growth conditions to a total thickness of $1.4 \mu \mathrm{m}$. The surface roughness and morphology are investigated by atomic force microscopy (AFM) and scanning tunneling microscopy (STM), both performed under ambient conditions in Park Scientific Instruments microscopes. The microstructural characterization (in plane view and cross-section) of the layer and of the interface is based upon conventional transmission electron microscopy in a Philips CM30, operated at $300 \mathrm{kV}$ and high resolution electron microscopy in a Philips CM300 UT, operated at $300 \mathrm{kV}$ (spherical aberration, $\mathrm{C}_{\mathrm{S}}=0.65 \mathrm{~mm}$, and a point-to-point resolution of $1.72 \AA$ ). The cross-sectional specimens

are prepared by conventional mechanical grinding and dimpling with diamond paste, followed by ion milling with $\mathrm{Ar}^{+}$ to electron transparency in a Gatan duomill (operated at $4.5 \mathrm{kV}, 1 \mathrm{~mA}, 13^{\circ}$, nitrogen cooling stage). Structural and chemical information is obtained from electron diffraction (SADP) (with high local resolution). The electro-optical analysis of our specimens consists of room temperature (300K) photoluminescence, produced by excitation with the $325 \mathrm{~nm}$ line of a HeCd-laser and cathodoluminescence at 77K. The PL intensities of the samples are collected in a double grating monochromator with a resolution of $0.8 \mathrm{~nm}$. The PL spectra are recorded in the wavelength range from $350 \mathrm{~nm}$ to $720 \mathrm{~nm}$. Typical PL spectra of $\alpha-\mathrm{GaN}$ exhibit emission in the ultraviolet range around $3.46 \mathrm{eV}$, the so called $\mathrm{I}_{2}$ line, which is attributed to the recombination of an exciton bound to a neutral donor [9]. Sharp PL lines are also observed around $3.36 \mathrm{eV}$ (the origin of which is not yet well understood). Moreover, a broad Gaussian-shaped luminescence band often consisting of several local maxima in the yellow region of the spectrum with a center at $2.2 \mathrm{eV}$ can be obtained, the origin of which is still under discussion. CL investigations are performed in a scanning electron microscope (SEM) of Cambridge Instruments, operated at 20keV at $77 \mathrm{~K}$ (with a spatial resolution of about $1.8 \mathrm{~nm}$ ) at beam currents between $15 \mathrm{nA}$ and $5 \mu \mathrm{A}$. CL complements to PL measurements (focus of laser beam limits spatial resolution) since they allow to probe with much higher local resolution and permit the relation of luminescent transitions to structural regions (and defects) in the specimen. To obtain CL images we map the photon energies around $528 \mathrm{~nm} / 2.35 \mathrm{eV}$ to locate regions responsible for the yellow luminescence on the specimen and around $358 \mathrm{~nm} / 3.46 \mathrm{eV}$ to localize regions belonging to the UV luminescence.

\section{Results}

We in the following compare two types of specimens, ' $Y$ ' and ' $B$ '. Figure 1 shows typical PL spectra of ' $Y$ ' ( Figure 1a) and ' $\mathrm{B}$ ' ( Figure 1b). Figure 2 shows the corresponding $C L$ spectra. The UV luminescence for the ' $\mathrm{B}$ ' sample is maximum at $358 \mathrm{~nm} / 3.46 \mathrm{eV}$ ( Figure $1 \mathrm{~b}$ ), belonging to the band-to-band transition. This observation is similar for the 'Y'sample.

The yellow luminescence in sample ' $\mathrm{B}$ ' in the form of a Gaussian shaped peak with the center at $528 \mathrm{~nm} / 2.35 \mathrm{eV}$ is very faint in PL ( Figure 1b) as well as in CL ( Figure 2b) measurements. This yellow luminescence in sample ' $Y$ ' however, is quite strong. The peak is Gaussian shaped in $\mathrm{CL}$ and PL with the center at $528 \mathrm{~nm} / 2.35 \mathrm{eV}$ and exhibits additional local maxima (cf. Figure 1 and Figure 2). CL investigations with different incident beam currents (cf. Figure 2a) show that the relative intensities depend on the beam current.

In the following we present investigations of the surface morphology and the structural properties of ' $\mathrm{Y}$ ' and ' $\mathrm{B}$ ' samples.

\subsection{Surface morphology}


Figure 3 shows the typical surface morphologies. Figure 3 a shows an STM micrograph giving the surface of an ' $Y$ ' sample; Figure $3 \mathrm{~b}$ shows an AFM micrograph of the same specimen with higher resolution. In ' $\mathrm{Y}$ ' samples

hexagonal islands are visible, which consist of 6 sideplane facets that intersect the (0001) surface in $\langle 10 \overline{1} 0\rangle$ directions. The typical island height is about $50 \mathrm{~nm}$ (peak to valley) at an average island width of $1-2 \mu \mathrm{m}$. The AFM image ( Figure $3 \mathrm{~b}$ ) is taken at the top of one of the hexagonal islands. The surface steps with the height of one unit cell originate from dislocations located in the center of the islands and having a Burgers vector component perpendicular to the surface. Figure 3a shows nanopipes, indicated by ' $\mathrm{N}$ ', with diameters greater than $50 \mathrm{~nm}$. These nanopipes occur in a density arround $10^{6} \mathrm{~cm}^{-2}$ and are exclusively found at the side facets of the hexagonal islands. Figure 3c shows an AFM micrograph of the surface of a ' $\mathrm{B}$ ' sample. An irregular rough surface with a typical roughness amplitude of about $150 \mathrm{~nm}$ at a similar wavelength is present. This roughness prevents the detection of nanopipes which are possibly present.

\subsection{Defect characterization}

Figure 4 and Figure 5 show typical cross-sectional TEM micrographs of samples ' $\mathrm{Y}$ ' and ' $\mathrm{B}$ '. A detailed defect characterization of both types of specimens gives the following results:

1. all dislocations propagate from the interface almost perpendicular to the surface, thus having $\langle 0001\rangle$ line directions;

2. close to the interface a very high density of dislocations with various Burgers vectors is present;

3. some of these dislocations react with each other or bend back to the interface, so that a reduced density of surviving dislocations is observed at a height larger than $400 \mathrm{~nm}$ above the interface.

The surviving dislocations are analyzed in the following.

Figure 4(a) and (b) show typical cross-sectional TEM micrographs of sample ' $Y$ '. Under the imaging conditions operating in Figure $4 \mathrm{a}$ all dislocations with a Burgers vector component in c-direction (i.e. dislocations of type $\vec{b}=[0001]$ and $\vec{b}=[\overline{1} \overline{1} 23]$ ) are visible. In Figure 4b imaging conditions are chosen such that dislocations with a Burgers vector $\vec{b}=[0001]$ are out of contrast and dislocations with Burgers vectors of type $\vec{b}=[\overline{1} 2 \overline{1} 0]$, i.e. dislocations with a Burgers vectors in the basal plane are visible. From a detailed contrast analysis we obtain a density of pure screw dislocations of type $\vec{b}=[0001]$ of $0.2 \cdot 10^{9} \mathrm{~cm}^{-2}$. The other dislocations having a Burgers vector component in the basal plane, i.e. dislocations of type $\vec{b}=[\overline{1} 2 \overline{1} 0]$ and $\vec{b}=[\overline{1} \overline{1} 23]$, occur in a density of $9.9 \cdot 10^{9} \mathrm{~cm}^{-2}$.

In Figure 5a and b typical cross-sectional TEM micrographs of sample 'B' are visible. A similar analysis as before yields a dislocation density of pure screw dislocations of type $\vec{b}=[0001]$ of $0.4 \cdot 10^{9} \mathrm{~cm}^{-2}$. Dislocations with a Burgers vector component in the basal plane have a density of $1.2 \cdot 10^{9} \mathrm{~cm}^{-2}$, of which $0.8 \cdot 10^{9} \mathrm{~cm}^{-2}$ lie on oblique planes and are of type $\vec{b}=[\overline{1} \overline{1} 23]$. Besides dislocations, a high density of stacking faults parallel to the interface is visible (cf. Figure 5). In the high resolution image in Figure 6(a) these planar defects are indicated by 'P'. Additional plane view investigations lead to the outline of these defects as sketched in Figure 6(b). These stacking faults are distributed all over the layer volume of ' $\mathrm{B}$ ' specimens. A fraction of the dislocations react with these planar defects.

A detailed analysis of the structure near the interface reveals $\alpha-G a N$ islands (grainlets) with the orientation $(0 \overline{1} 11$ ) GaN parallel to (0001) $\mathrm{Al}_{2} \mathrm{O}_{3}$ (cf. Figure 7). These grainlets have a width and height of about 10nm, an average separation of about $20 \mathrm{~nm}$ and turn out to be evidence of a novel misfit strain relaxation mechanism. This mechanism and related finite element calculations of grainlet-induced strain relaxation are described in detail elsewhere [15]. In the present case, grainlet-misfit relaxation is $82 \%$ of the total geometrical misfit.

\subsection{Correlation between surface morphology, defect structure and electro-optical data}

Figure 8 shows TEM plane view images of specimen ' $Y$ '. Under the imaging conditions used, screw dislocations with a Burgers vector $\vec{b}=[0001]$ are out of contrast. Only dislocations with a Burgers vector component in the 
basal plane are visible $(b=1 / 3[1210])$ and $b=1 / 3[1123])$. From Figure 8a it is obvious that the hexagonal surface morphology (cf. Figure 3a) is reproduced by the arrangement of dislocations, i.e. small angle grain boundaries are present between the hexagonal islands as already observed and suggested by Ponce et al. [9] and Ning et al. [16]. Inside these islands planar defects are visible that lie perpendicular to the surface and in blocks of three form stars with $120^{\circ}$ between pairs. No dislocation with a Burgers vector component in the interface

is visible in these islands. Under the imaging conditions of Figure $8 \mathrm{~b}$ additional dislocations of type $\vec{b}=[0001]$ are visible as dark lines. These dislocations are randomly distributed in the complete layer volume. This finding of randomly distributed screw dislocations inside the islands corroborates the AFM observations (cf. Figure $3 a b$ which shows a distribution of screw dislocation induced growth spirals at the surface).

A comparison to $\mathrm{CL}$ mappings of the surface taken at different wavelengths points to remarkable features. Figure 9 a shows a micrograph taken in the light of UV $(258 \mathrm{~nm} / 3.46 \mathrm{eV})$. Figure $9 \mathrm{~b}$ shows the same area taken with secondary electrons. It is evident that the regions of strong UV luminescence (bright areas in Figure 9a) correspond to the troughs in the surface morphology. Using the yellow line at $528 \mathrm{~nm} / 2.35 \mathrm{eV}$ or others from the yellow group (cf. Figure 2), a homogeneous distribution is seen, thus the images are not shown. In summary for the sample ' $Y$ ': UV luminescence essentially originates from the trough areas, while the yellow luminescence is observed at all sites on the surface. In sample ' $\mathrm{B}$ ' the CL intensity distribution, both in yellow and in UV lines, is inhomogeneous over the surface but unspecific and not correlated with the surface morphology.

\section{Discussion}

In the following we will focus on the correlation between the volume defect structure and the CL/PL findings.

Therefore we first summarize the differences in structural properties between sample ' $Y$ ' and 'B'. First, the density of dislocations with a Burgers vector component in the interface (i.e. $\vec{b}=[\overline{1} 2 \overline{1} 0]$ or $\vec{b}=[\overline{1} \overline{1} 23]$ type dislocations), in the following called 'edge-type dislocations' is one order of magnitude larger in sample ' $Y$ ' than in sample ' $B$ '. Second, in sample ' $Y$ ' the density of pure screw dislocations (i.e. $\vec{b}=[0001]$ ) is larger by a factor of 3 in sample ' $Y$ ' than in sample 'B' $\left(0.4\right.$ versus $\left.1.3 \cdot 10^{9} \mathrm{~cm}^{-2}\right)$. If we now assume that the dislocations contribute notably to the strong yellow luminescence in sample ' $\mathrm{Y}$ ', two possibilities have to be considered: a higher density of screw dislocations only in sample ' $Y$ ' or the higher total dislocation density.

The high dislocation density in all GaN samples complicates a direct correlation between dislocation type and CL data because of the limited resolution in the CL-SEM, where no single dislocations can be distinguished, especially at high dislocation densities. This can easily be seen at sample 'B' where CL shows an inhomogeneous, unspecific UV and yellow luminescence which cannot be correlated to individual defects (all different types of dislocations are statistically distributed in the whole layer). The analysis is facilitated in sample ' $Y$ ', where edge-type dislocations are confined to small angle grain boundaries, while screw dislocations are still statistically distributed. Here we can correlate UV luminescence with troughs and thus with the presence of edge-type dislocations that are confined to the troughs. Yellow luminescence is distributed over the whole specimen as are the screw dislocations. On the other hand, 'edge-type' dislocations do not significantly influence the yellow luminescence, as otherwise troughs would show up clearly in the yellow luminescence CL map. A possible explaination for this may be that 'edge-type" dislocations induce only shallow levels in the bandgap, while screw dislocations may form deep levels. At a first look this contradicts Ponces [9], who in his work relates the yellow luminescence to small angle grain-boundaries at hexagonal islands. However, it does not become clear from Ponces' paper which type of dislocations form these small angle grain boundaries. The small angle grain boundaries observed by Ponce might well contain a pronounced amount of screw dislocations that according to our results cause yellow luminescence. According to Ning et al. [16] such small angle grain boundaries may form due to growth of columnar grains.

\section{Conclusions}

Our correlation between volume defect structure and the spectrally and locally resolved CL measurements can be interpreted as follows:

1. screw dislocations (i.e. $\vec{b}=[0001]$ ) essentially contribute to yellow luminescence;

2. 'edge-type' dislocations (i.e. $\vec{b}=[\overline{1} 2 \overline{1} 0]$ or $\vec{b}=[\overline{1} \overline{1} 23]$ ) do favour luminescence and not impair band-to-band transition. 


\section{References}

[1] Shuji Nakamura, Takashi Mukai, Masayuki Senoh, Appl. Phys. Lett. 64, 1687-1689 (1994).

[2] I. Akasaki, H. Amano, J. Cryst. Growth 146, 455 (1995).

[3] K. G. Fertitta, A. L. Holmes, F. J. Ciuba, R. D. Dupuis, F. A. Ponce, J. Electron. Mater. 24, 257 (1995).

[4] M. Kamp, M. Mayer, A. Pelzmann, A. Thies, H. Y. Chung, H. Sternschulte, O. Marti, K. J. Ebeling, Mater. Res. Soc. Symp. Proc. 395, 135-139 (1996).

[5]"Thermally cracked $\mathrm{NH}_{3}$ for growth of GaN", M. Kamp, M. Mayer, A. Pelzmann, S. Menzel, H. Y. Chung, H. Sternschulte, K. J. Ebeling, in: Proc. of Topical Workshop on III/V Nitrides, Nagoya, Japan (1995)

[6] R. C. Powell, N.-E. Lee, Y.-W. Kim, J. E. Greene, J. Appl. Phys. 73, 189-204 (1993).

[7] J. I. Pankove, J. E. Berkeyheiser, H. P. Maruska, J. Wittke, Sol. St. Comm. 8, 1051 (1970).

[8] R. Dingle, M. llegems, Sol. St. Comm. 9, 175 (1971).

[9] F. A. Ponce, D. P. Bour, W. Gotz, P. J. Wright, Appl. Phys. Lett. 68, 57-59 (1996).

[10] T. Ogino, M. Aoki, Jpn. J. Appl. Phys. 19, 2395 (1980).

[11] P. Perlin, T. Suski, H. Teisseyre, M. Leszczynski, I. Grzegory, J. Jun, S. Porowski, P.Boguslawski, J. Bernholc, J. C. Chervin, A. Polian, T. D. Moustakas, Phys. Rev. Lett. 75, 296-299 (1995).

[12] F. A. Ponce, D. P. Bour, W. Gotz, N. M. Johnson, H. I. Helava, I. Grzegory, J. Jun, S. Porowski, Appl. Phys. Lett. 68, 917-919 (1996).

[13] E. R. Glaser, T. A. Kennedy, K. Doverspike, L. B. Rowland, D. K. Gaskill , J. A. Freitas, Jr., M. Asif Khan, D. T. Olson, J. N. Kuznia, D. K. Wickenden, Phys. Rev. B 51, 13326-13336 (1995).

[14] R. Singh, R. J. Molnar, M. S. Unlu, T. D. Moustakas, Appl. Phys. Lett. 64, 336-338 (1994).

[15] M. Albrecht, S. Christiansen, H. P. Strunk, unpublished (1996).

[16] X. J. Ning, F. R. Chien, P. Pirouz, J. W. Yang, M. Asif Khan , J. Mater. Res. 11, 580-592 (1996). 


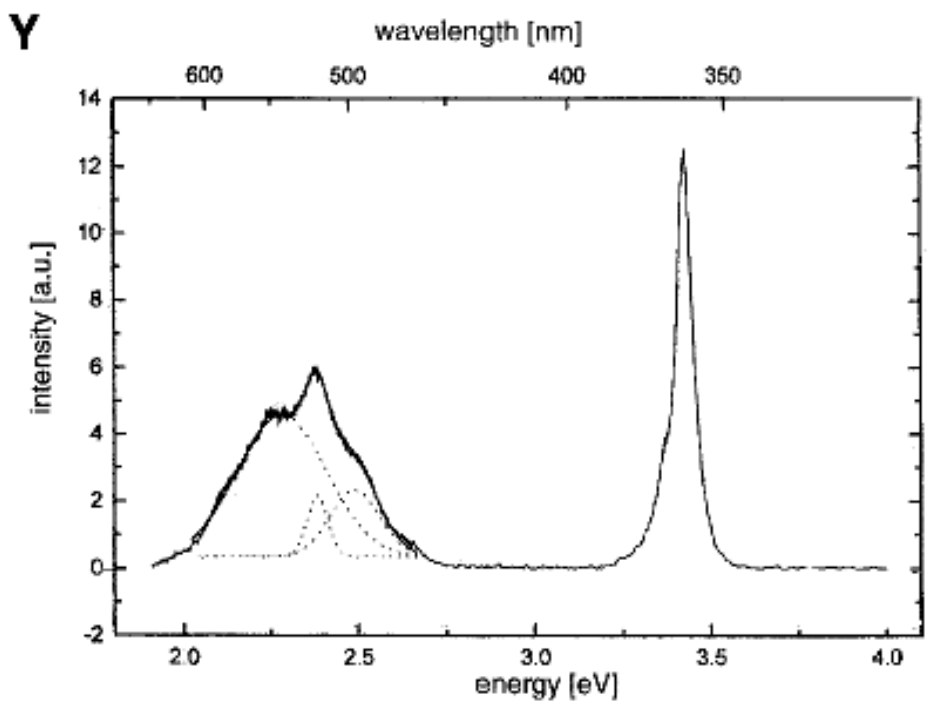

a

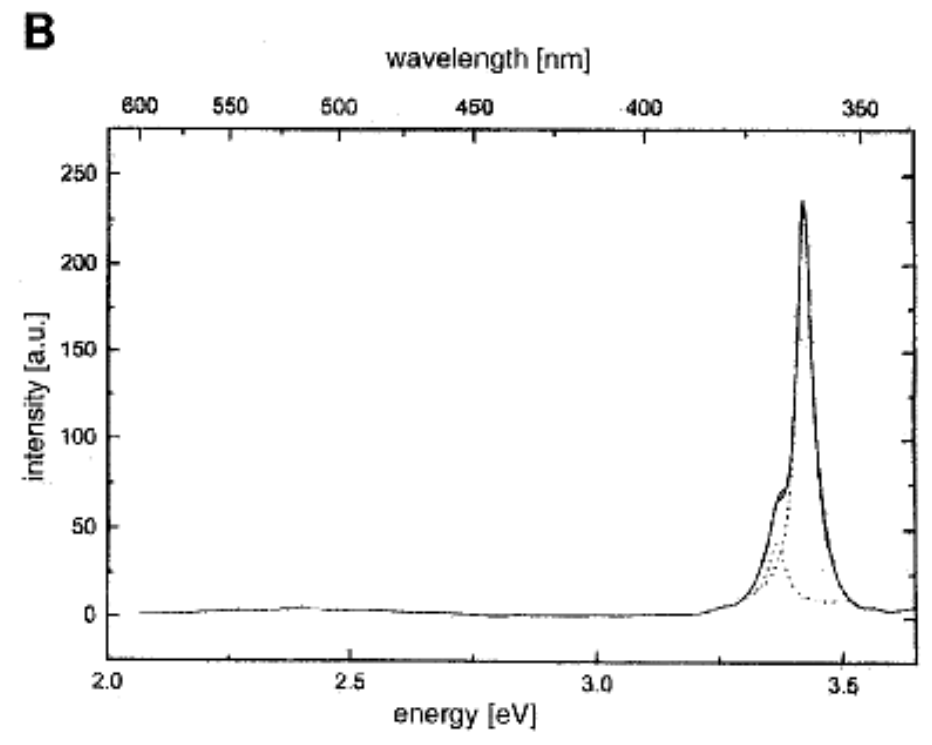

b
Figure 1. Photoluminescence spectra taken at 300K of: (a) $1.7 \mu \mathrm{m}$ thick GaN layer of sample ' $Y$ '. A UV luminescence peak at $358 \mathrm{~nm} / 3.46 \mathrm{eV}$ and a split yellow luminescence peak with the center at $528 \mathrm{~nm} / 2.35 \mathrm{eV}$ are distinguished; (b) $1.4 \mu \mathrm{m}$ thick GaN layer of sample 'B'. A UV luminescence peak at $358 \mathrm{~nm} / 3.46 \mathrm{eV}$ and only a very faint yellow luminescence peak at $528 \mathrm{~nm} / 2.35 \mathrm{eV}$ are distinguished. 


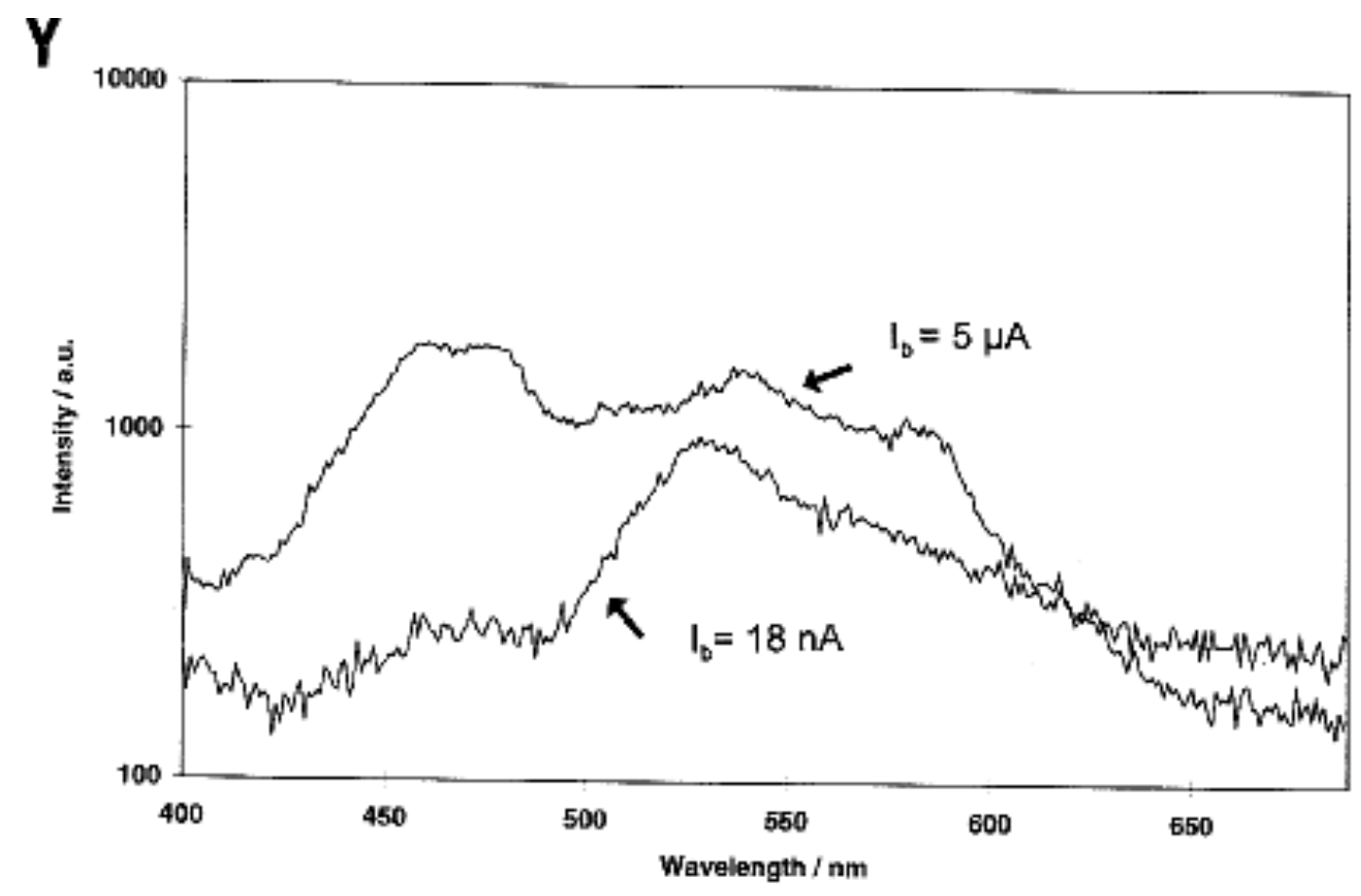

a

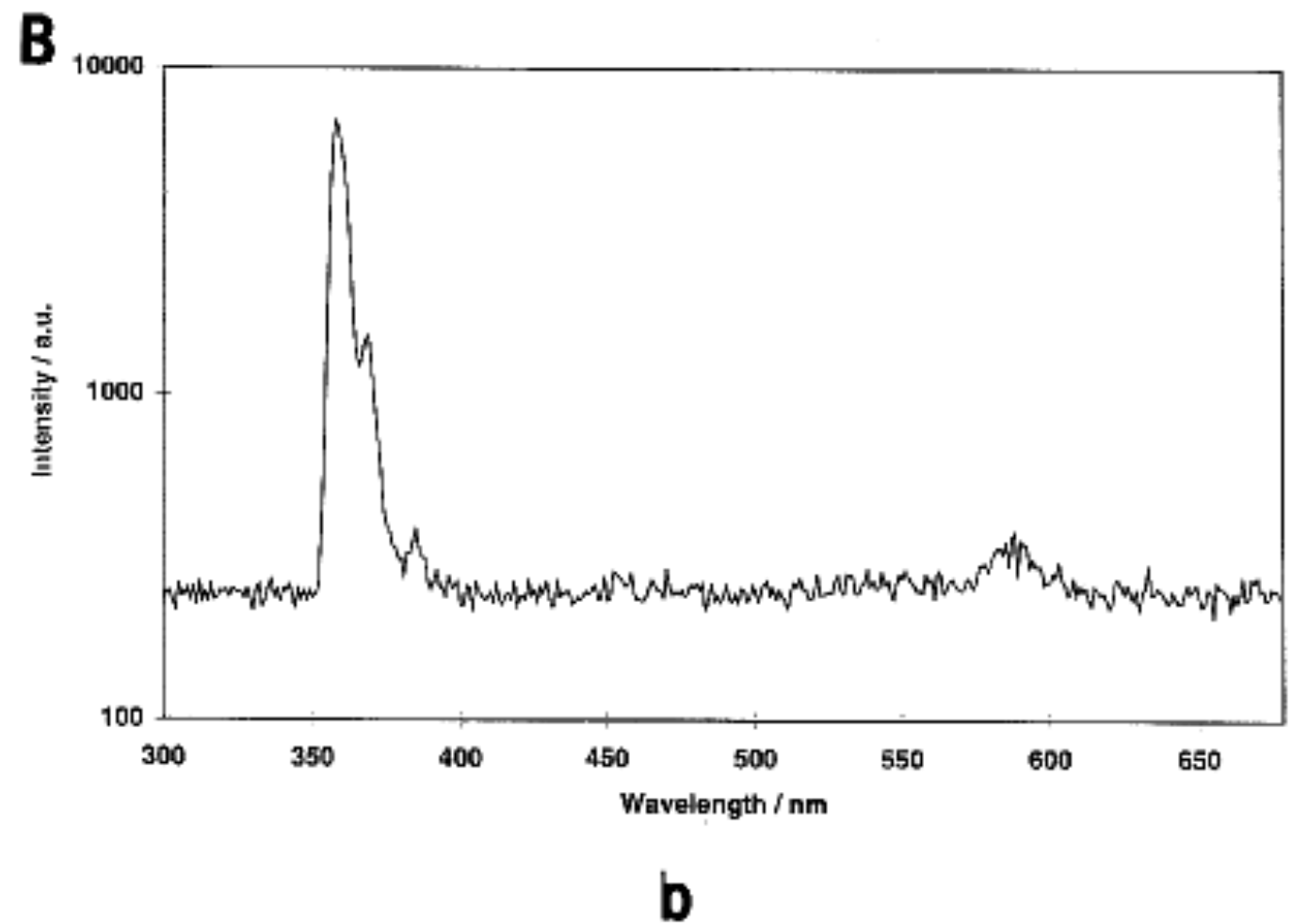

Figure 2. Cathodoluminescence spectra taken at $77 \mathrm{~K}$, electron beam energy $20 \mathrm{keV}$, beam current $0.17 \mu \mathrm{A}$, area of measurement $10 \mu \mathrm{m} \times 10 \mu \mathrm{m}$. The dotted lines represent descriptions using Gaussian peaks. (a) Sample ' $Y$ ': only the longer (yellow) wavelength range is shown to exhibit the dependenc of the spectrum on the beam current. The strong CL peak centered at $358 \mathrm{~nm} / 3.46 \mathrm{eV}$ is omitted. (b) Sample 'B': strong UV luminescence, very faint yellow $\mathrm{CL}$ signal. 


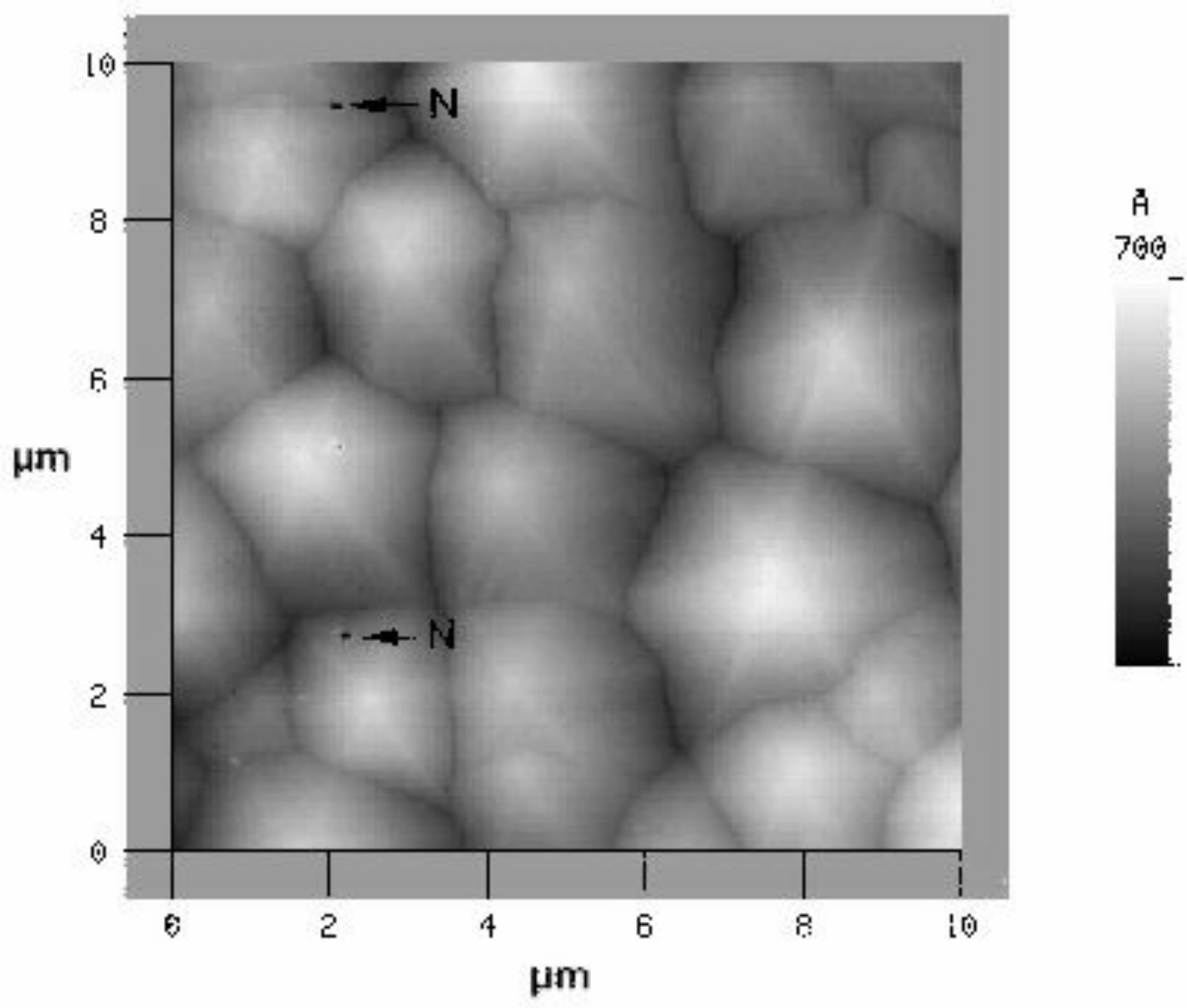

Figure 3a. Surface morphology of a ' $Y$ ' sample: facetted islands with a typical width of $1-2 \mu \mathrm{m}$ and a typical height of about $50 \mathrm{~nm}$; nanopipes, which are located on sideplane facets are indicated by ' $\mathrm{N}$; STM, $10 \mu \mathrm{m} \times 10 \mu \mathrm{m}$; 


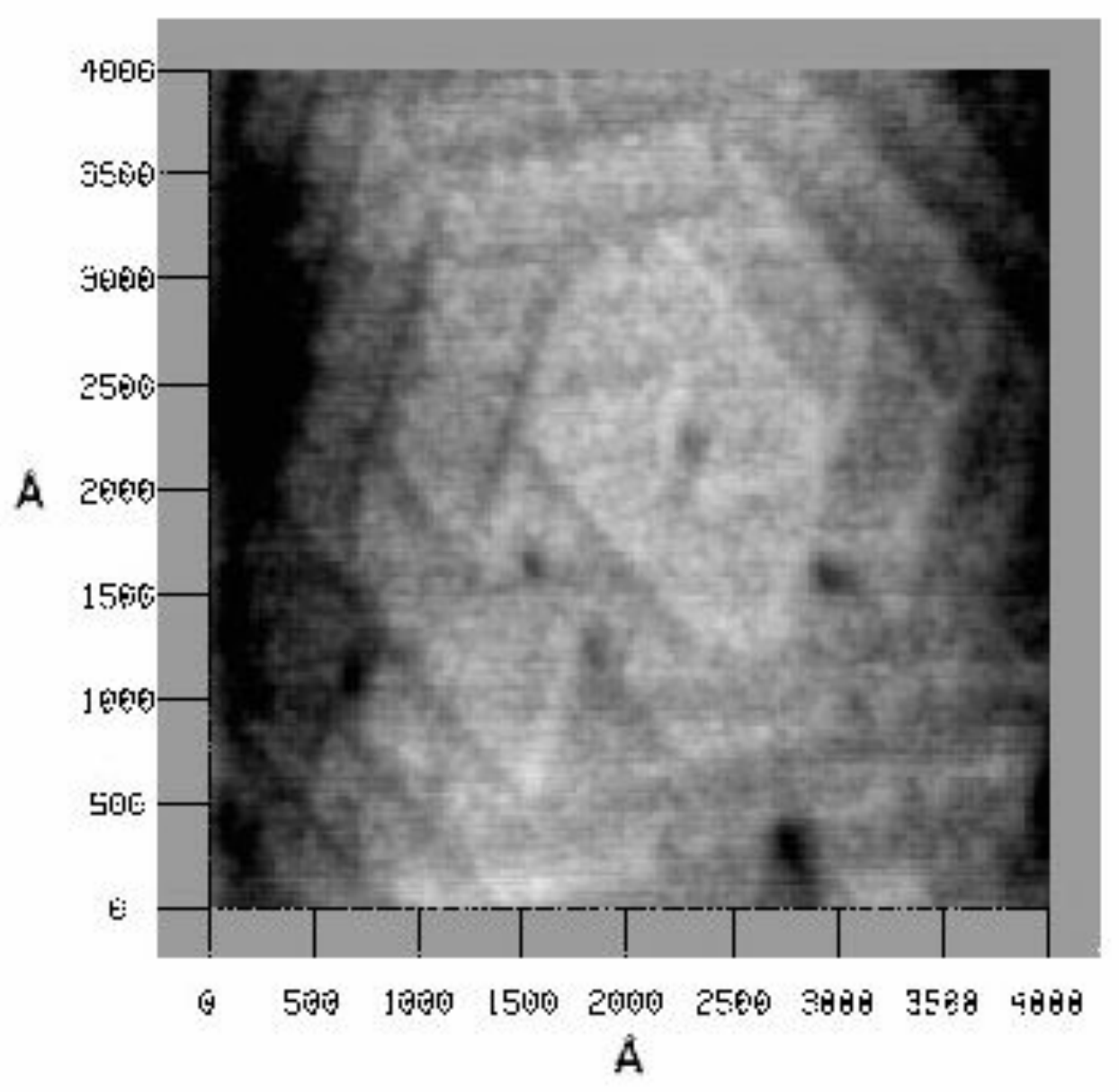

Figure $\mathbf{3 b}$. Top of such a facetted island. A stepped surface formed by hexagonal growth spirals is visible; AFM, $0.4 \mu \mathrm{m} \times 0.4 \mu \mathrm{m}$ 


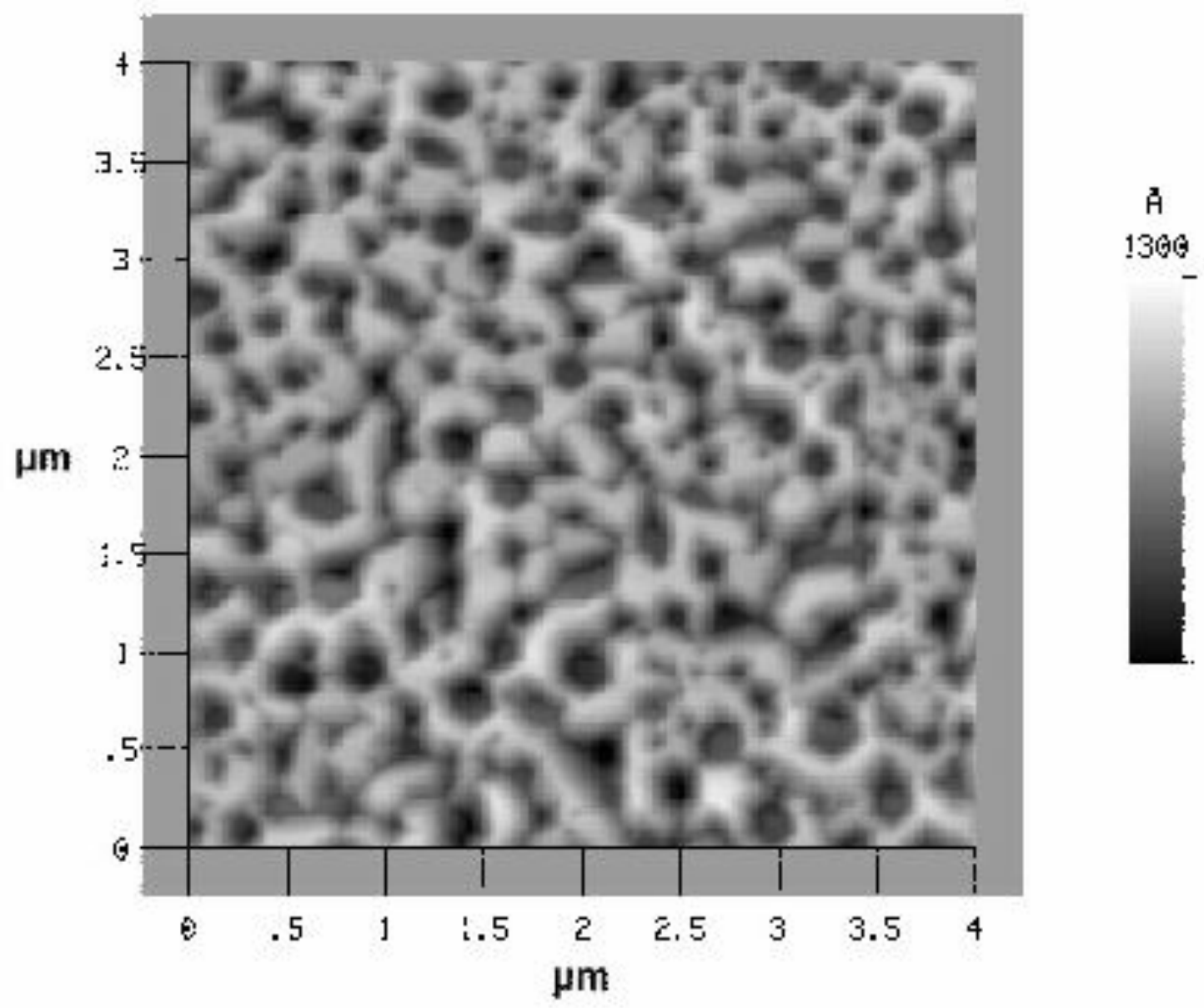

Figure 3c. Surface morphology of a 'B' sample: an irregular rough surface with a typical roughness amplitude of about $150 \mathrm{~nm}$ at a similar width is visible; AFM, $4 \mu \mathrm{m} \times 4 \mu \mathrm{m}$; 

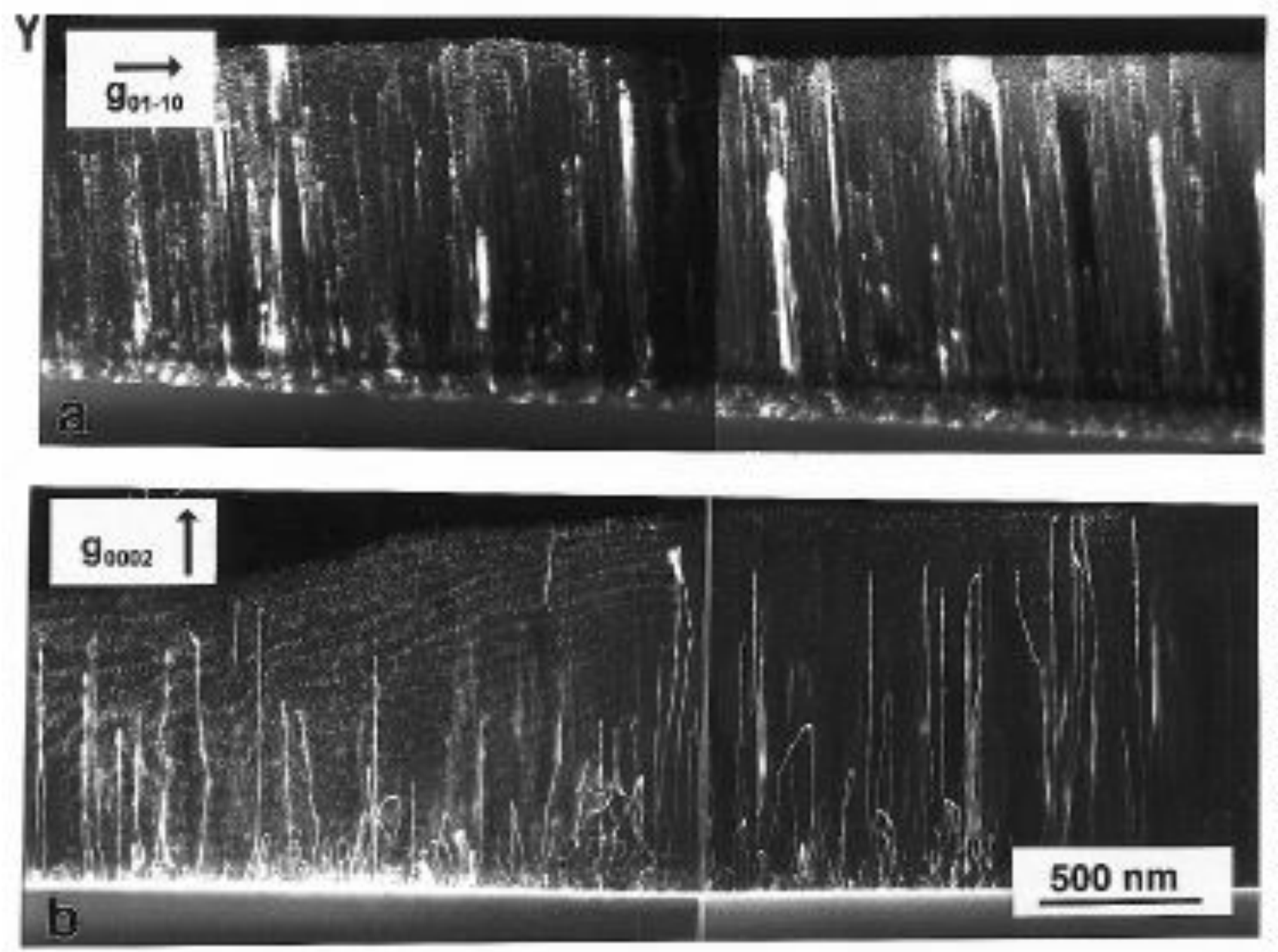

Figure 4. Dislocation arrangement in the ' $Y$ ' sample: (a) screw dislocations with $\vec{b}=[0001]$ are out of contrast, dislocations with $a \vec{b}$-component in the interface, i.e. $\vec{b}=1 / 3[\overline{1} 2 \overline{1} 0]$ and $\vec{b}=1 / 3[\overline{1} 2 \overline{1} 0]$ are visible; cross-sectional TEM $(\vec{g} / 3 \vec{g})$ weak beam image, $\vec{g}=(01 \overline{1} 0) ;($ b) screw dislocations with $\vec{b}=[0001]$ are visible; dislocations with a $\vec{b}$-component in the interface are out of contrast; cross-sectional TEM weak beam image $(\vec{g} / 3 \vec{g}), \vec{g}=(0002)$; 

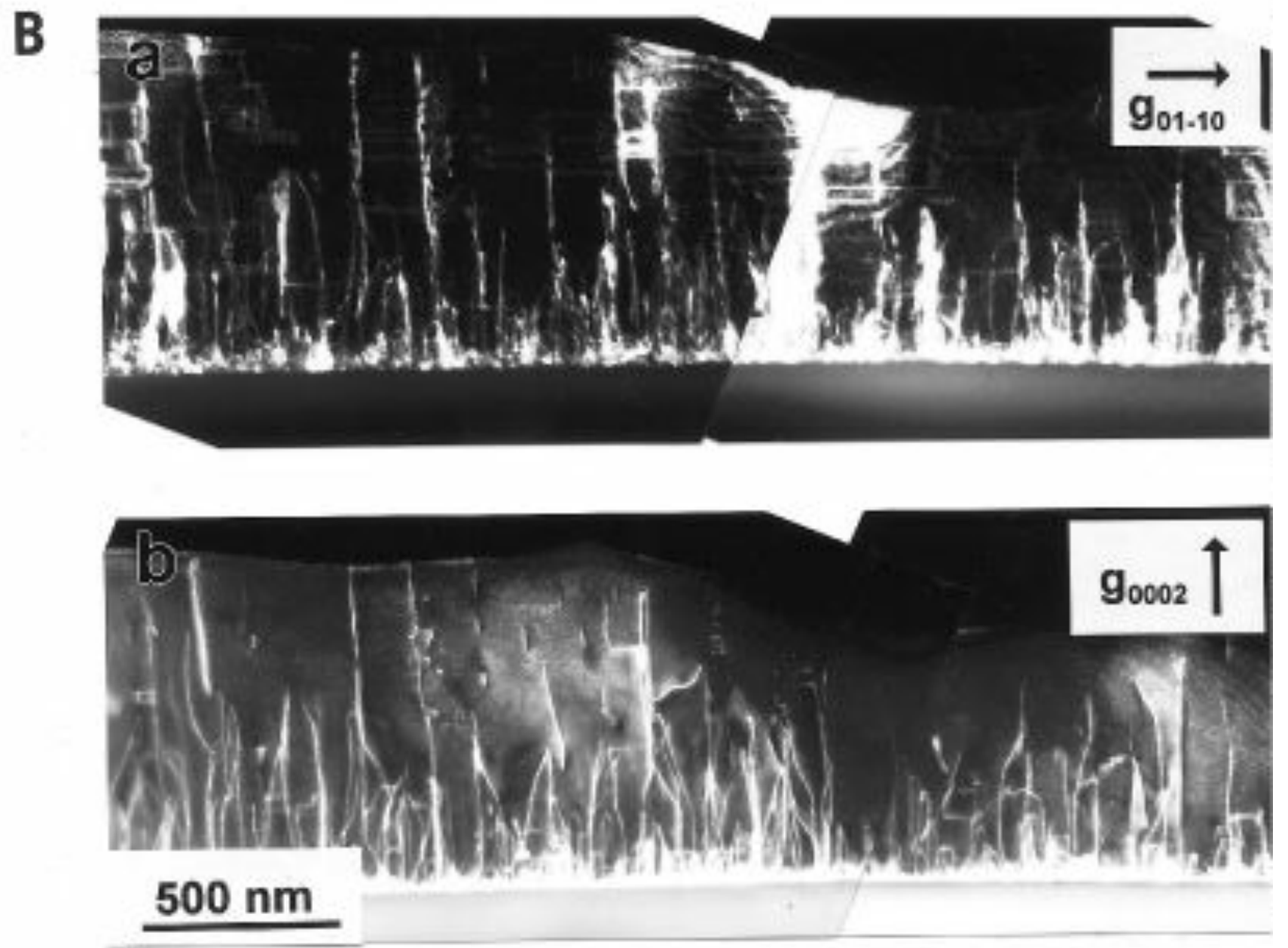

Figure 5. Dislocation arrangement in the 'B' sample: (a) screw dislocations with $\vec{b}=[0001]$ are out of contrast, misfit dislocations with a $\vec{b}$-component in the interface $(\vec{b}=1 / 3[\overline{1} 2 \overline{1} 0], \vec{b}=1 / 3[\overline{1} 2 \overline{1} 0]$ ) are visible; cross-sectional TEM weak beam image $(\vec{g} / 3 \vec{g}), \vec{g}=(01 \overline{1} 0) ;($ b) screw dislocations with $\vec{b}=[0001]$ are visible; misfit dislocations with a $\vec{b}$-component in the interface are out of contrast; cross-sectional TEM weak beam image $(\vec{g}(3 \vec{g}), \vec{g}=(0002)$; 
a

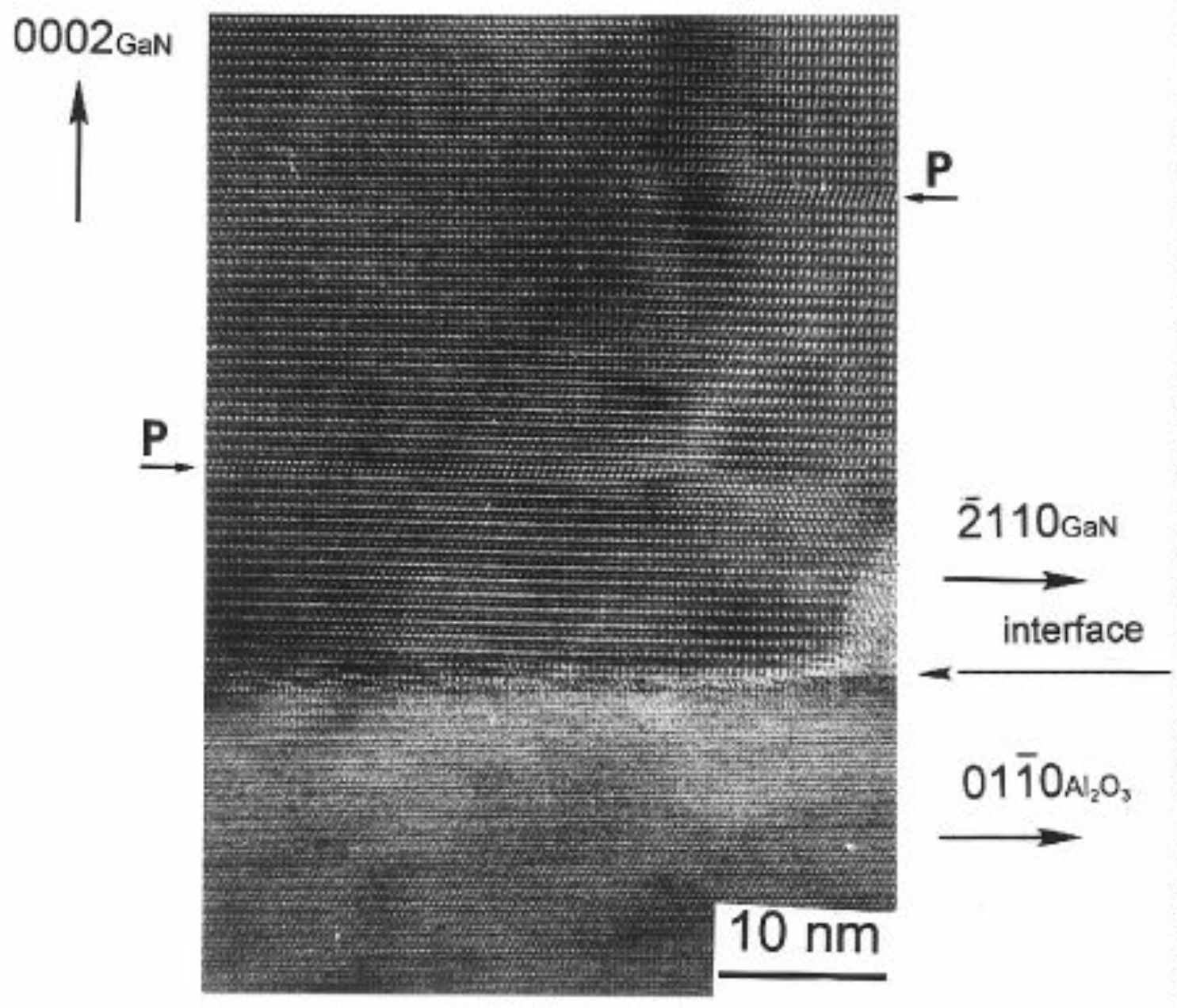

b

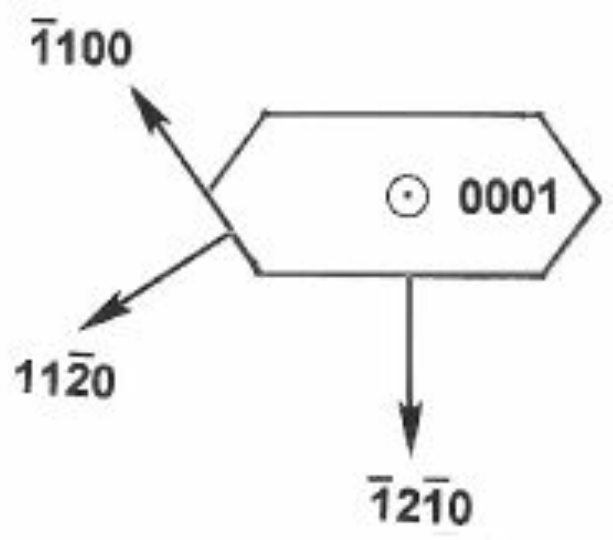

Figure 6. (a) Planar defects, lying parallel to the interface are indicated by ' $P$ '. High resolution micrograph from the layer/substrate interface region of sample ' $\mathrm{B}$ '. The incident beam is along the $[01 \overline{10}]_{\mathrm{G} \text { aN }}$ zone axis; cross-section (XTEM); (b) Schematic top view (parallel to the [0001]-direction) representation of a planar defect. 

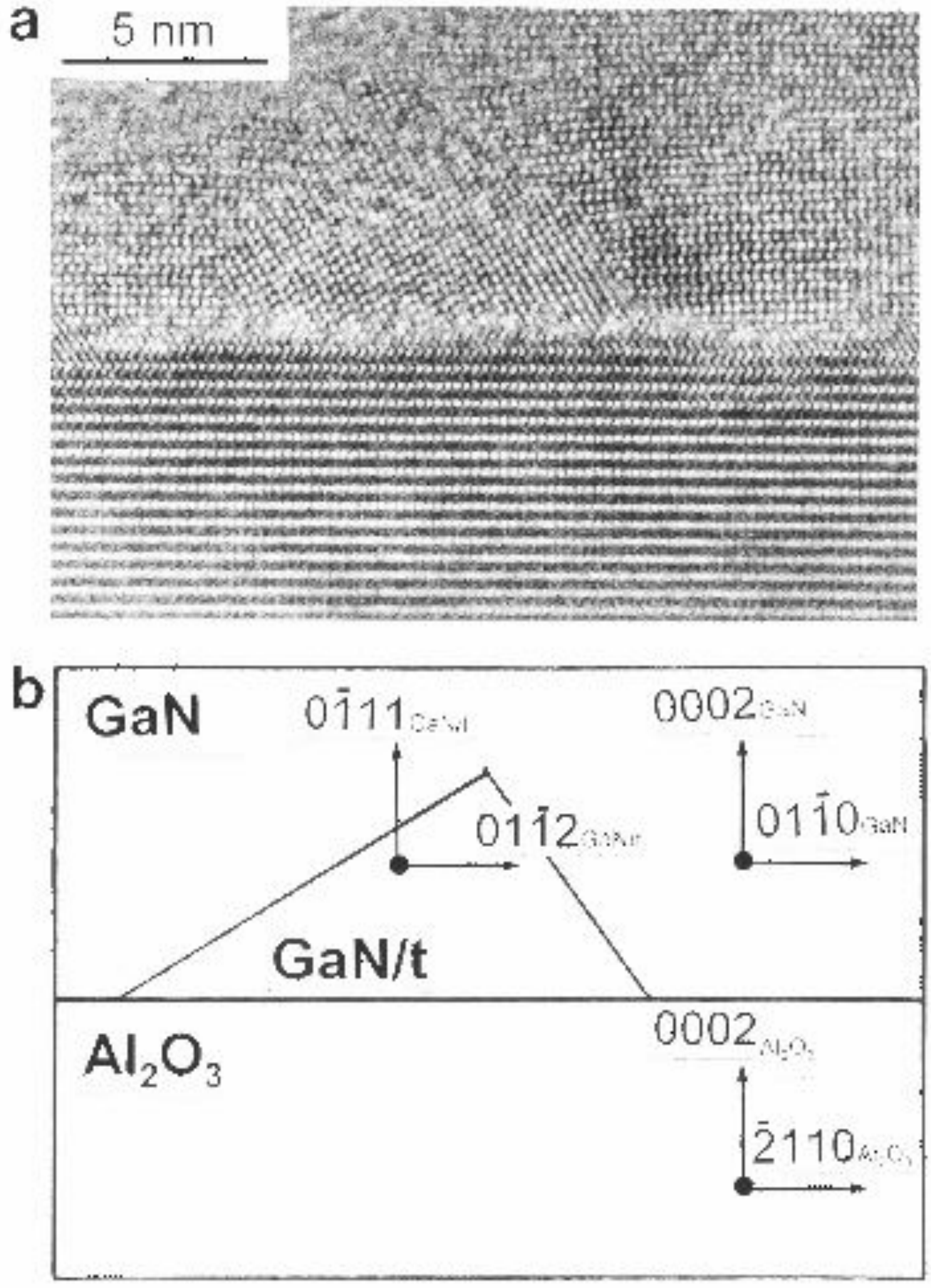

Figure 7. (a) 'Grainlets' at the layer/substrate interface with an epitaxial orientation shown in (b). High resolution micrograph of sample ' $\mathrm{B}$ '. The incident beam is along the $[\overline{2} 110] \mathrm{GoN}$ zone axis; XTEM; (b) Schematic representation of the grainlet-misfit relaxation configuration at the interface. 

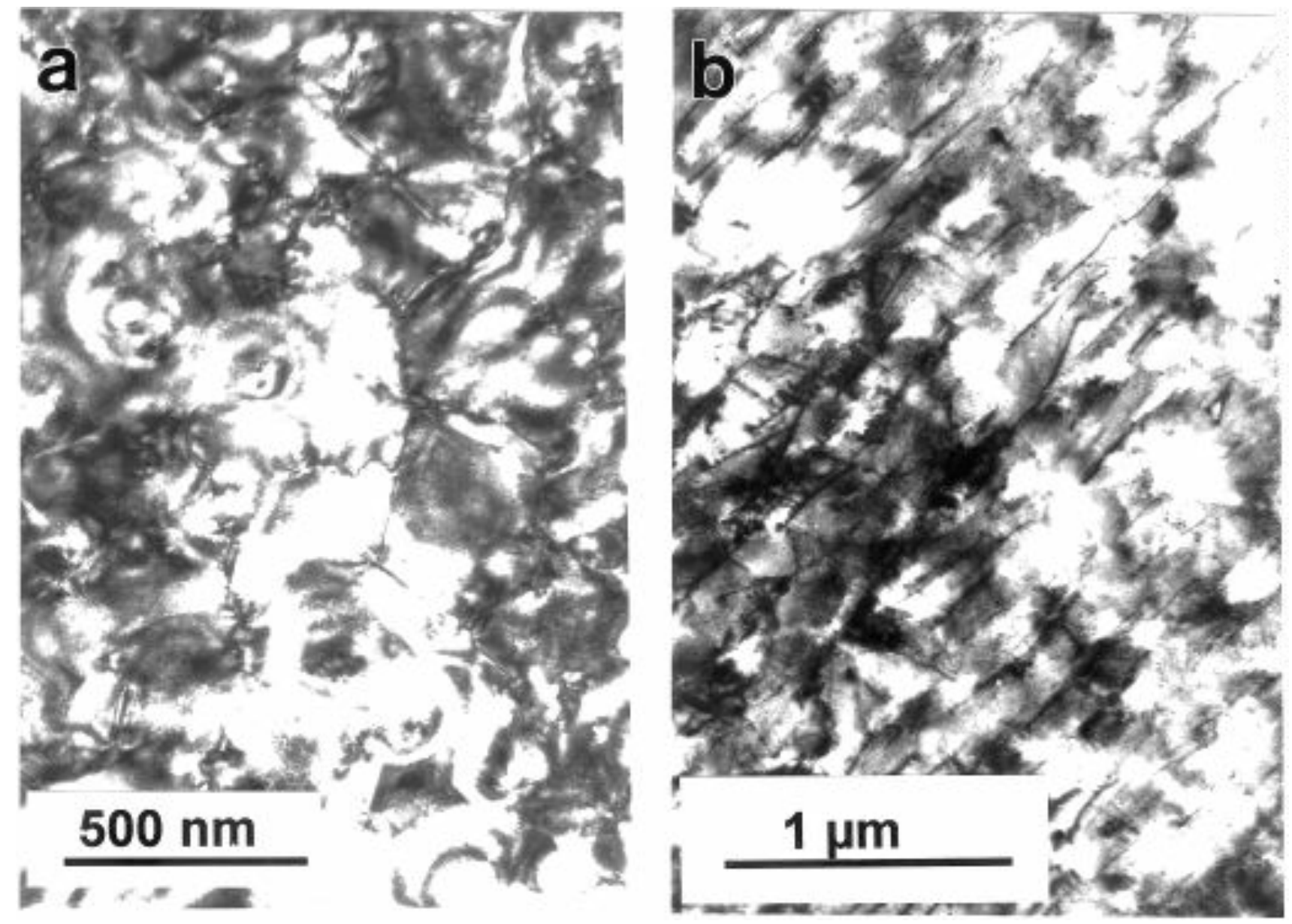

Figure 8. Plane-view micrographs taken from sample ' $Y$ '. (a) the hexagonally faceted islands (cf. Figure 3a) are visible with small angle tilt boundaries formed by edge-type dislocations at the rims between adjacent hexagonal islands; these boundaries lie perpendicular to the interface; inside the islands planar defects perpendicular to the interface, are visible; these planar defects in blocks of three form stars with $120^{\circ}$ between pairs; multi- beam conditions, incident beam parallel to the [0001]-zone axis; $(b) \vec{b}=[0001]$ dislocations, randomly distributed in the complete layer volume, are visible as dark lines; two-beam conditions, $\vec{g}=(01 \overline{1} 1)$; 


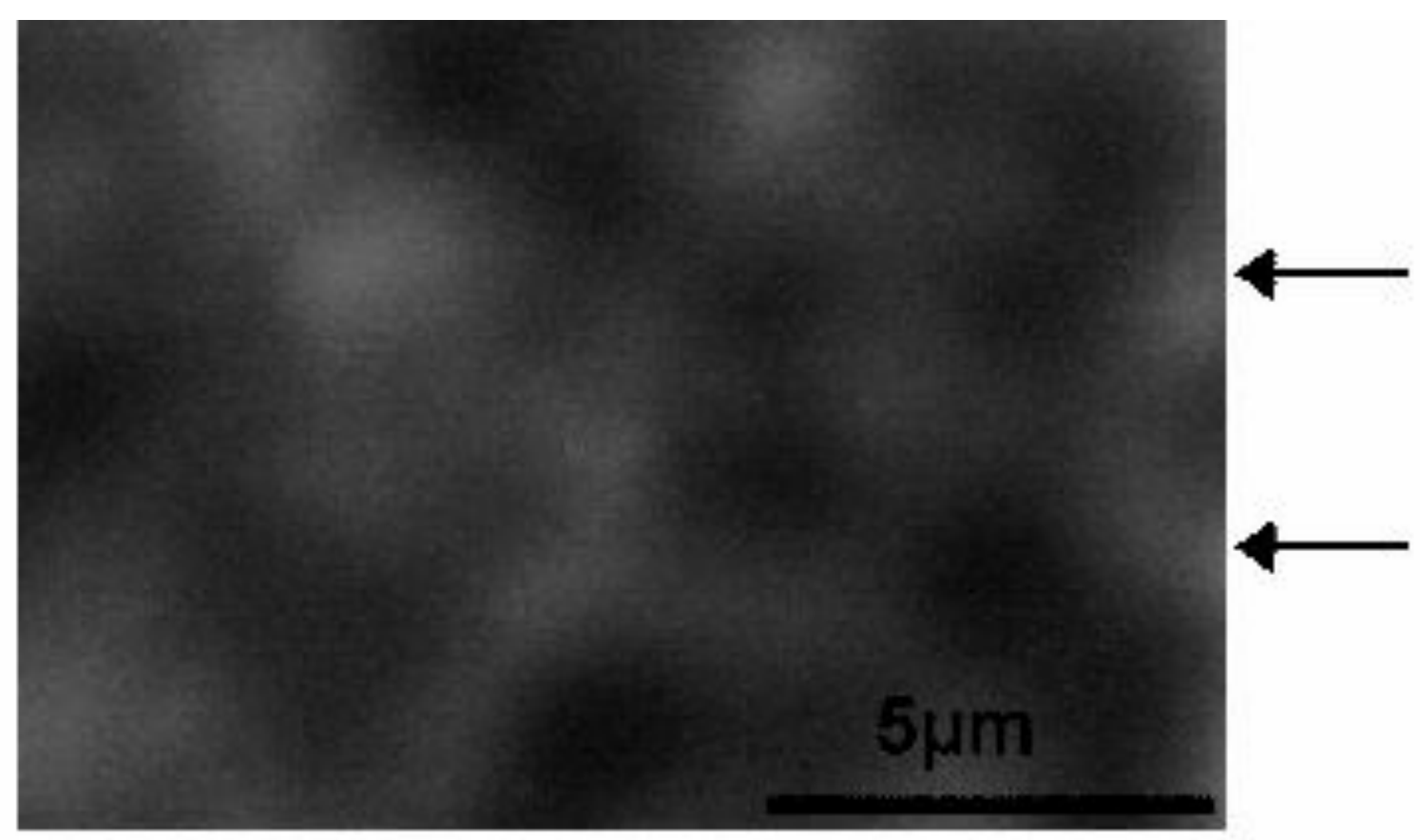

\section{$358 \mathrm{~nm} / 3.46 \mathrm{eV}$}

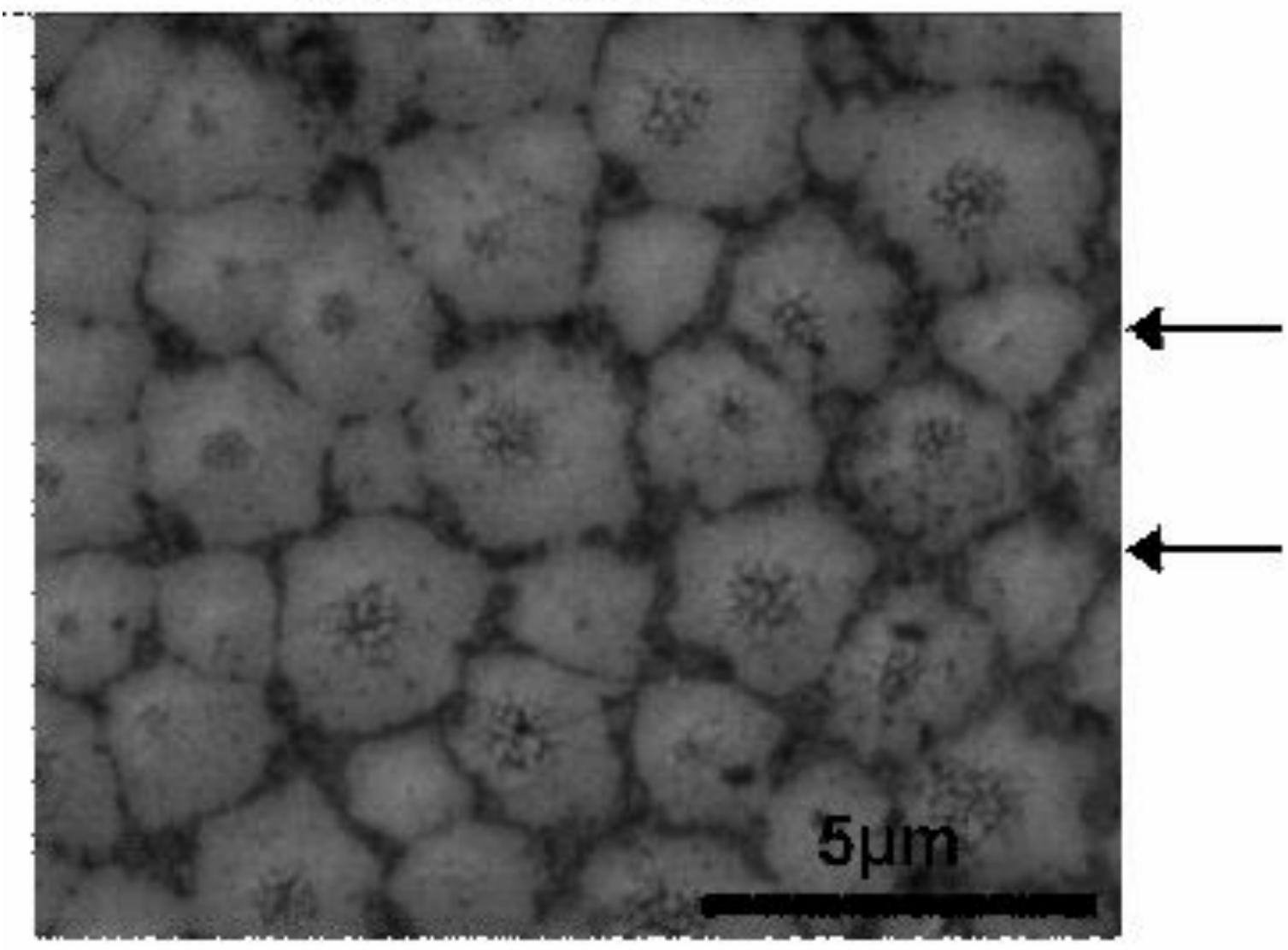

Figure 9. CL mappings of ' $Y$ ' specimen: (a) image taken at $358 \mathrm{~nm} / 3.46 \mathrm{eV}$ (UV luminescence); the troughs between adjacent islands are visible in bright contrast, i.e. the UV luminescence is confined to these troughs; (b) secondary electron image, obtained at $20 \mathrm{keV}$ of the same region on the specimen.

(C) 1996-1997 The Materials Research Society 


\begin{tabular}{|c|c|c|c|c|}
\hline M & R & $\mathrm{S}$ & Internet Journal of & Nitride Semiconductor Research \\
\hline
\end{tabular}

